\title{
Low Molecular Weight Dextran in Experimental Pancreatitis: Effects on Pancreatic Microcirculation
}

\author{
James A. KNOl, M.D., LeSlie P. EDGComb, M.D., MaRK G. InMan, M.S., \\ AND FREDERIC E. ECKHAUSER, M.D.
}

Surgical Research Laboratories, Veterans Administration Medical Center, Ann Arbor, Michigan 48105, and Department of Surgery, University of Michigan Medical Center, Ann Arbor, Michigan 48109

Presented at the Annual Meeting of the Association for Academic Surgery,

San Diego, California, November 7-10, 1982

\begin{abstract}
Although low molecular weight (LMW) dextran has been said to decrease the lethality of experimental acute pancreatitis (AP) by reversing stasis in the pancreatic microcirculation, the actual mechanism(s) of action is unknown. This investigation was designed to mcasure the effects of low molecular weight dextran on pancreatic capillary flow $\left(Q_{\mathrm{CAP}}\right)$ and arteriovenous shunt flow $\left(Q_{\mathrm{Avs}}\right)$, and on pancreatic oxygen consumption $\left(\mathrm{O}_{2} C_{\mathrm{P}}\right)$ following bile-trypsin-induced AP in dogs. Total pancreatic blood flow $\left(Q_{\mathrm{T}}\right)$ was measured with an electromagnetic flow probe on the superior pancreaticoduodenal artery (SPDA). $Q_{\text {Avs }}$ was measured by liver trapping of ${ }^{99 \mathrm{~m}} \mathrm{Tc}$-albumin microspheres after SPDA injection. $Q_{\text {CAP }}$ was calculated as $Q_{\tau}$ minus $Q_{\text {Avs }}$. Seventeen dogs were treated with lactated Ringer's (LR) solution at $6.5 \mathrm{ml} / \mathrm{kg} / \mathrm{hr} ; 10$ dogs were treated with LMW dextran $10 \%$ in normal saline at $1.5 \mathrm{ml} / \mathrm{kg} / \mathrm{hr}$ plus LR at $5.0 \mathrm{ml} / \mathrm{kg} / \mathrm{hr}$. Mean arterial and central venous pressures remained constant throughout the 4hr experiment. In the dogs receiving LR only, $Q_{\mathrm{T}}$ decreased from 42.7 to $24.4 \mathrm{ml} / \mathrm{min}(P<0.001)$; $Q_{\text {Avs }}$ remained constant at $1.35 \pm 0.04 \mathrm{ml} / \mathrm{min}$. During the first $30 \mathrm{~min} \mathrm{O}_{2} C_{\mathrm{p}}$ decreased from 1.17 to $0.76 \mathrm{ml} \mathrm{O}_{2} / \mathrm{min}(P<0.05)$ and remained constant thereafter. LMW dextran treatment altered none of these hemodynamic or metabolic parameters significantly. Conclusions: bile trypsin AP in the dog causes significant decreases in $Q_{\mathrm{T}}$ and $Q_{\mathrm{CAP}}$ without altering $Q_{\mathrm{Avs}}$. The decrease in $\mathrm{O}_{2} C_{\mathrm{P}}$ in association with a constant $Q_{\text {Avs }}$ suggests a metabolic block to oxygen uptake at the cellular level. Continuous infusion of LMW dextran at a dose of $1.5 \mathrm{ml} / \mathrm{kg} / \mathrm{hr}$ in the dog does not reverse these abnormalities.
\end{abstract}

\section{INTRODUCTION}

Since Panum showed in 1862 that acute pancreatitis could be produced by injection of wax droplets into the pancreatic arterial circulation, it has been recognized that vascular damage may play a role in acute pancreatitis [22]. Increasingly, there has been emphasis on the interplay of the "vascular factors" in the pathological progression of simple edematous pancreatitis to frank parenchymal necrosis. Studies of pancreatic histopathology after pancreatitis have shown damage to blood vessel walls, the presence of venous thrombi [28], and poor filling of vessels in intralobular areas [29]. Severe constriction of pancreatic arteries and arterioles as well as an increase in the size and number of arteriovenous shunts and severe venous stasis have also been observed [24].
Clinically it was recognized that severe fluid volume deficits were characteristic of acute hemorrhagic pancreatitis and that vigorous fluid replacement improved survival [10]. Dextrans were first evaluated as therapeutic agents in acute pancreatitis because of their ability to expand intravascular volume, but initial trials were disappointing $[2,19]$. However, subsequent experimental studies were more encouraging. Animals treated with low molecular weight (LMW) dextran showed improved survival compared to those treated with albumin or crystalloid solutions [7, 32]. In addition, evidence accumulated that pancreatic blood flow was maintained in animals with hemorrhagic pancreatitis with the use of LMW dextran $[6,8,15]$. In contrast, salinetreated animals demonstrated markedly decreased pancreatic blood flow. Moreover, in certain types of experimental pancreatitis, 
LMW dextran has also been shown to have salutary effects on pancreatic oxygen consumption [9].

Work by Donaldson and Schenk suggested increased intrapancreatic arteriovenous (AV) shunting as the etiology of the decreased pancreatic oxygen consumption seen with acute pancreatitis [9]. Increased AV shunting in acute pancreatitis has ncver been documented, and the effect of LMW dextran on such shunts is not known. This study was undertaken (1) to investigate the changes in pancreatic blood flow distribution between the capillary circulation and through arteriovenous shunts; (2) to determine whether the observed decreases in pancreatic oxygen consumption occur primarily from increased arteriovenous shunt flow; and (3) to determine the influence of LMW dextran on both the distribution of pancreatic blood flow and on pancreatic oxygen consumption.

\section{MATERIALS AND METHODS}

Twenty-seven conditioned mongrel dogs of both sexes, weighing 20.9 to $47.7 \mathrm{~kg}$, were fasted for $20 \mathrm{hr}$, anesthetized with intravenous thiopental sodium $(30 \mathrm{mg} / \mathrm{kg})$, intubated, and ventilated with room air using a Harvard ventilator. Anesthesia was maintained with intermittent supplemental doses of Pentothal sodium. Respiratory activity was adjusted to maintain a slight hypocarbia and a normal $P_{2}$. The animal's core temperature was maintained at $38 \pm 0.5^{\circ} \mathrm{C}$ with a heating mattress and heat lamps. The left internal jugular vein was cannulated for fluid administration and central venous pressure monitoring. The right internal jugular vein was cannulated with a Swan-Ganz catheter for thermodilution cardiac output studies and for mixed venous blood sampling. The right carotid artery was cannulated for pressure monitoring and arterial blood sampling.

Preliminary studies in our laboratory have confirmed the compartmental nature of the arterial blood supply to the canine pancreas. The superior pancreaticoduodenal artery supplies the head, neck, and most of the uncinate process, the splenic artery provides branches supplying the body and tail, and the inferior pancreaticoduodenal artery supplies the distal tip of the uncinate process. A method commonly used to measure pancreatic blood flow employs an electromagnetic flow probe placed around the gastroduodenal artery after ligation of the right gastroepiploic artery, the pancreatic branches of the splenic artery, and the inferior pancreaticoduodenal artery [8]. However, we have documented significantly decreased perfusion of the body and tail when the splenic artery branches to the pancreas are ligated. To measure blood flow through a single inflow vessel and to exclude changes which might result from hypoperfusion of a segment of the pancreas, our model included ligation of the pancreas at the neck and excision of the body and tail, with recording of the weight of the excised segment (Fig. 1). The inferior pancreaticoduodenal artery was ligated with preservation of its accompanying vein. Paraduodenal collaterals running along the distal duodenum were also ligated. The right gastroepiploic artery was ligated distally and cannulated proximally, with the tip of the cannula positioned at the junction with the gastroduodenal artery. To obtain pancreatic effluent blood for oxygen analysis, the superior pancreaticoduodenal vein was cannulated at a point distal to its pancreatic tributaries, and the tip of the cannula was positioned at a point proximal to all pancreatic tributaries, but short of its junction with the portal vein. The major pancreatic duct was cannulated external to the duodenum. The duct from the uncinate process was cannulated either at its bifurcation with the major duct or at the level of the accessory duct. When not cannulated, the accessory duct was ligated. An electromagnetic flow probe was placed around the gastroduodenal artery distal to the last hepatic artery branch and an encircling snare was placed on the gastroduodenal artery proximal to the flow probe for purposes of intermittent occlusion.

Following the surgical procedure, the animals were allowed to stabilize for $30 \mathrm{~min}$. Two sets of baseline measurements were then re- 


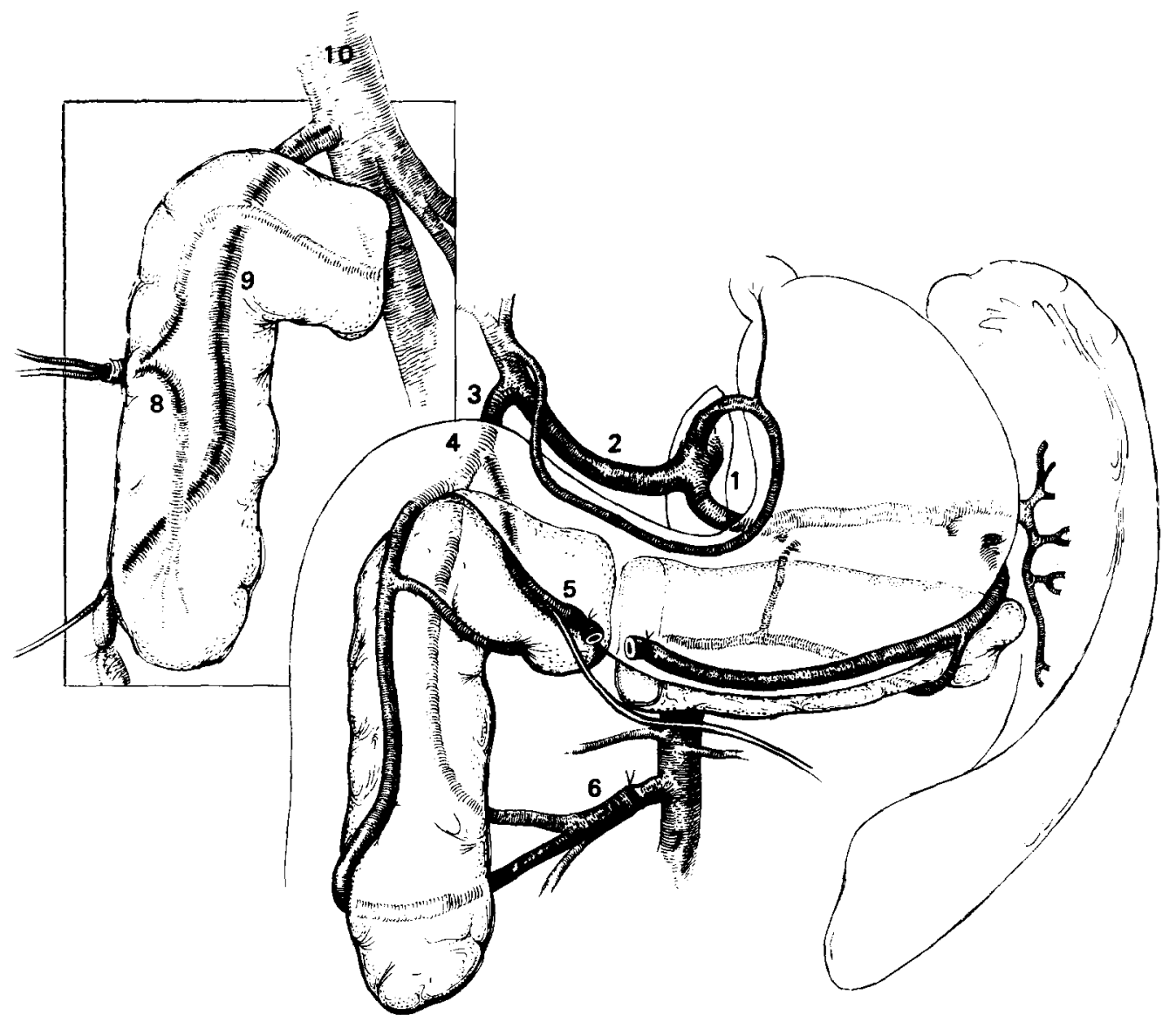

FIG. 1. Pancreatitis model: the body and tail of the pancreas have been excised, the right gastroepiploic artery (5) has been cannulated, and the inferior pancreaticoduodenal artery (6) has been ligated. Inset: The superior pancreaticoduodenal vein (9) and the pancreatic ducts (8) have been cannulated. The venous cannula has been placed with its tip just short of the portal vein (10).

corded at $30-\mathrm{min}$ intervals. Pancreatitis was induced by high pressure injection $(140 \mathrm{~mm}$ $\mathrm{Hg}$ ) of a solution of sterile autogenous bile (1 $\mathrm{ml} / \mathrm{kg}$ ) containing crystalline trypsin $(2000 \mathrm{U} /$ $\mathrm{ml}$ bile) [21].

Arteriovenous shunting across the pancreas was estimated by measurement of liver trapping of $15-25-\mu \mathrm{m}^{99 \mathrm{~m}}$ Tc-labeled albumin microspheres (3M) injected into the pancreatic arterial circulation via the right gastroepiploic artery cannula. During injection, the snare on the gastroduodenal artery was tightened to prevent retrograde flow of microspheres into the hepatic arteries. Liver trapping of microspheres was measured by a gamma probe directed transversely at the right lobe of the liver through the right chest wall, using a lead shield to exclude activity from the pancreas. Standardization was obtained at the termination of the experiment by injection of two doses of labeled microspheres directly into the portal vein via the superior pancreaticoduodenal venous catheter. The proportion of each injected dose passing through the pancreas to the liver was taken as a measure of percent AV shunting.

One group (LR) of 17 dogs received lactated Ringer's solution at $6.5 \mathrm{ml} / \mathrm{kg} / \mathrm{hr}$. Five dogs were observed for $3 \mathrm{hr}$ and 12 dogs were observed for $4 \mathrm{hr}$. A second group (DEX) of 10 dogs received LMW dextran (Rheomacrodex), $10 \%$ solution in normal saline, at 1.5 $\mathrm{ml} / \mathrm{kg} / \mathrm{hr}$, supplemented by lactated Ringer's solution at $5.0 \mathrm{ml} / \mathrm{kg} / \mathrm{hr}$. The dextran infu- 
sion was begun with the induction of pancreatitis. In the DEX group one dog was observed for $3 \mathrm{hr}$ and 9 dogs were observed for $4 \mathrm{hr}$. Measurements were taken at 30-min intervals. At the termination of the experiment the animals were sacrificed, the remaining pancreas was excised and weighed, and samples were prepared for histologic evaluation.

Statistical analysis utilized Student's $t$ test for parametric data and the median test for nonparametric data. Analysis was based both on changes from initial, using each dog as its own control, and on absolute values. Variance is expressed as standard error of the mean.

\section{RESULTS}

Hemorrhagic pancreatitis developed in all animals. Observed changes in gross morphology included diffuse hemorrhage into and around the gland, saponification of surrounding fat, and accumulation of significant amounts of hemorrhagic fluid within the abdominal cavity. In preliminary studies, this appearance correlated closely with the histologic picture of early necrotizing pancreatitis.

Systemically, few alterations were observed. Arterial pressure and central venous pressure did not vary significantly from baseline, and there were no significant differences between the experimental and control groups of animals (Fig. 2). Cardiac index was decreased in both groups of dogs (Fig. 2). The LR dogs showed decreases that were significant $(P<0.05)$ at all time points beyond 60 min, with a maximal decrease of $22 \%$ from a baseline value of $2.79 \pm 0.18$ to $2.17 \pm 0.18$ liters $/ \mathrm{min}^{-\mathrm{m}^{2}}$ at $3 \mathrm{hr}$. By comparison, the DEX dogs showed a lesser maximal decrease (12\%) from $2.89 \pm 0.19$ to $2.50 \pm 0.13$ liters/ min- $\mathrm{m}^{2}$, not a statistically significant decrease. Differences in cardiac index between the groups were not statistically significant. Systemic oxygen extraction, when correlated with time, increased significantly in both groups $(P<0.05)$ over the $4 \mathrm{hr}$ of the experiment, but there were no consistent significant intergroup differences (Fig. 3).

Following the induction of pancreatitis,

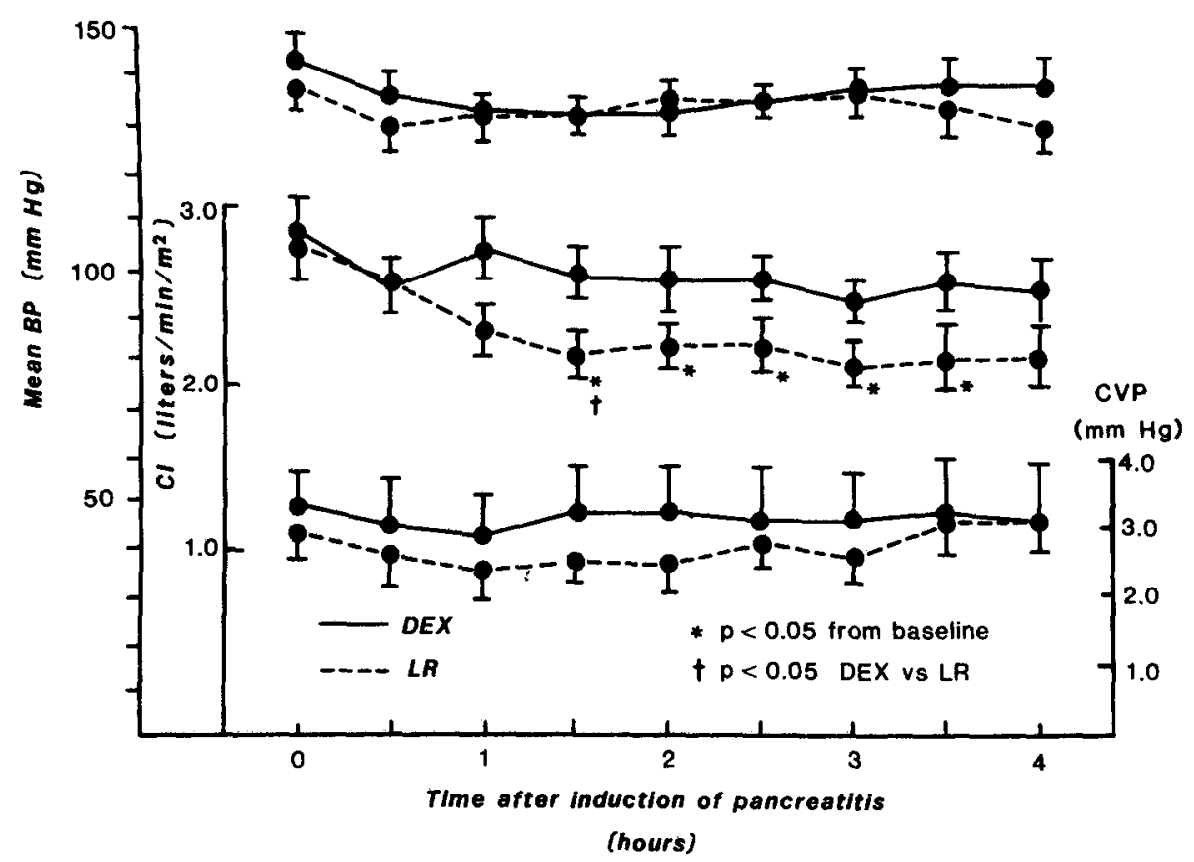

FIG. 2. Alterations in systemic blood pressure (BP), cardiac index (CI), and central venous pressure (CVP) following induction of pancreatitis. 


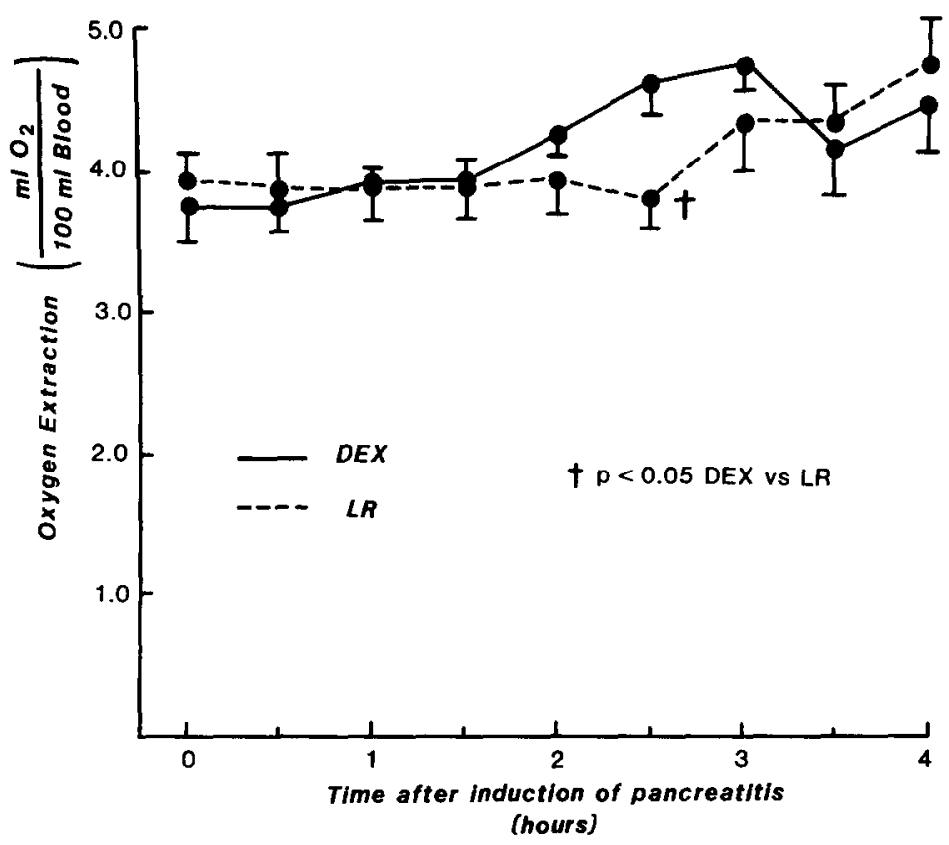

FIG. 3. Systemic oxygen extraction following induction of pancreatitis.

pancreatic arterial blood flow decreased in the LR dogs from a baseline value of $42.7 \pm 3.3$ $\mathrm{ml} / \mathrm{min}$ to $33.9 \pm 2.7 \mathrm{ml} / \mathrm{min}$ at $60 \mathrm{~min}$, and continued to decline to $24.9 \pm 1.7 \mathrm{ml} / \mathrm{min}$ at $3 \mathrm{hr}$ (Fig. 4). The maximal decrease in pancreatic blood flow (43\%) to $24.4 \pm 2.2 \mathrm{ml} /$ min occurred at $4 \mathrm{hr}$. DEX dogs showed a similar maximal decrease (42\%) from 62.7 $\pm 6.5 \mathrm{ml} / \mathrm{min}$ at baseline to $36.3 \pm 5.3 \mathrm{ml} /$ min at $3.5 \mathrm{hr}$. Although there appeared to be significant intergroup differences in pancreatic blood flow, those differences were present prior to the induction of pancreatitis. Comparison of the groups using each animal as its own control demonstrated no significant differences in the relative decreases in flow between the groups (Fig. 5). Comparison of changes in pancreatic blood flow relative to systemic blood flow (pancreatic flow/cardjac output) showed a decline in both groups, but the observed changes were not significant (Fig. 5). Arteriovenous shunt flow remained nearly constant between 1 and $2 \mathrm{ml} / \mathrm{min}$ throughout the experimental period, and no intergroup differences were observed (Fig. 4).

Pancreatic oxygen consumption decreased within $\mathbf{3 0}$ min after induction of pancreatitis from $1.17 \pm 0.14$ to $0.76 \pm 0.11 \mathrm{ml} \mathrm{O}_{2} / \mathrm{min}$ $(P<0.05)$ in the LR group, and from 1.60 \pm 0.15 to $0.73 \pm 0.09 \mathrm{ml} \mathrm{O} / \min (P<0.01)$ in the DEX group (Fig. 6). Following the initial fall, there was no significant further decrease over the ensuing $3.5 \mathrm{hr}$; in addition, there were no significant intergroup differences.

\section{DISCUSSION}

The hemodynamic changes which accompany acute pancreatitis have long been of interest. Historically, systemic changes resulting from acute pancreatitis were the primary focus of attention. Local changes occurring in pancreatic hemodynamics have elicited increasing attention, as it has become more apparent that the prcaression of pancreatitis from a benign reversible condition to one of pancreatic necrosis may be mediated by local vascular changes. That these vascular factors may be of significance has been shown experimentally in models where acute hemorrhagic pancreatitis was induced by the injection of particulate material into the pancreatic arterial circulation. Worsening pancreatitis appeared 


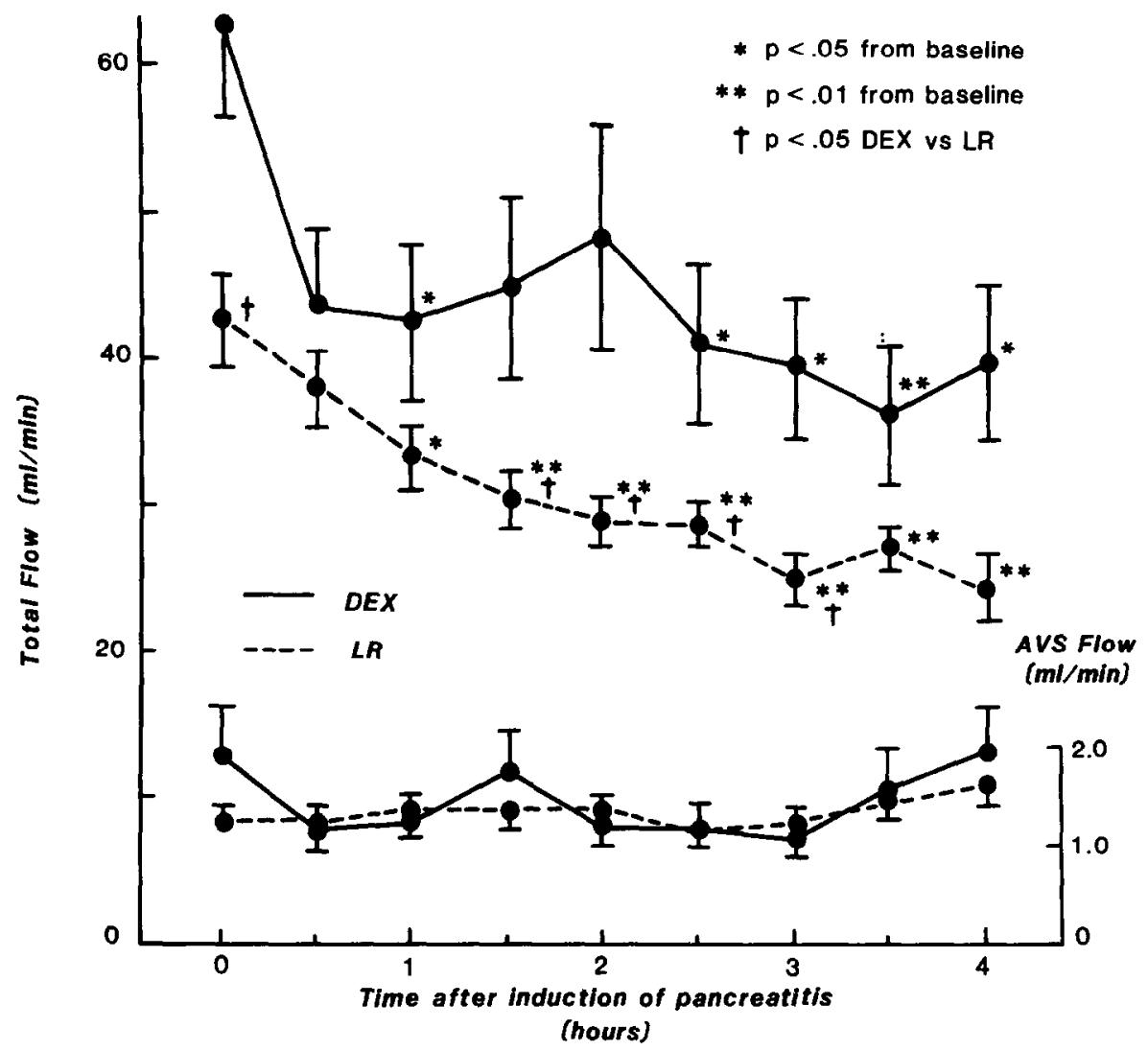

FIG. 4. Total pancreatic blood flow and arteriovenous shunt flow following induction of pancreatitis.

to develop in proportion to the extent of blockage of the pancreatic microcirculation [25]. It has also been shown that edematous pancreatitis can be transformed to hemorrhagic pancreatitis by brief temporary occlusion of the arterial supply [27], or by occlusion of the pancreatic venous drainage $[1,3]$.

Several investigators have demonstrated that pancreatic blood flow decreases after induction of acute pancreatitis. Early studies utilized the ${ }^{86} \mathrm{Rb}$-distribution method [15, 23]. A subsequent study utilizing venous outflow measurements confirmed findings of decreased pancreatic blood flow in acute experimental pancreatitis [26]. Recently, using electromagnetic flow monitoring, the finding was further supported $[8,20]$. It is also of some interest that investigators have demonstrated that pancreatic blood flow decreases out of proportion to observed decreases in cardiac output following experimental pancreatitis $[8,15]$.
It has been suggested that the decreased pancreatic blood flow observed in acute pancreatitis is due to a primary alteration in pancreatic microcirculatory dynamics. Another alternative is that decreases in pancreatic blood flow may be secondary to decreased systemic flow (cardiac output) and simply reflect changes seen elsewhere in the body. We have previously demonstrated a decrease in pancreatic blood flow $3 \mathrm{hr}$ after induction of acute pancreatitis, despite administration of intravenous fluids at a rate sufficient to maintain cardiac index and CVP; no similar changes in pancreatic blood flow were seen in animals without acute pancreatitis [20]. These findings were confirmed in this study where, in spite of efforts to keep systemic changes to a minimum, significant decreases in pancreatic blood flow were seen in both groups of dogs (Figs. 4, 5). The observed decreases in the ratio of pancreatic flow to cardiac output (Fig. 5 ), though not statistically significant, further 


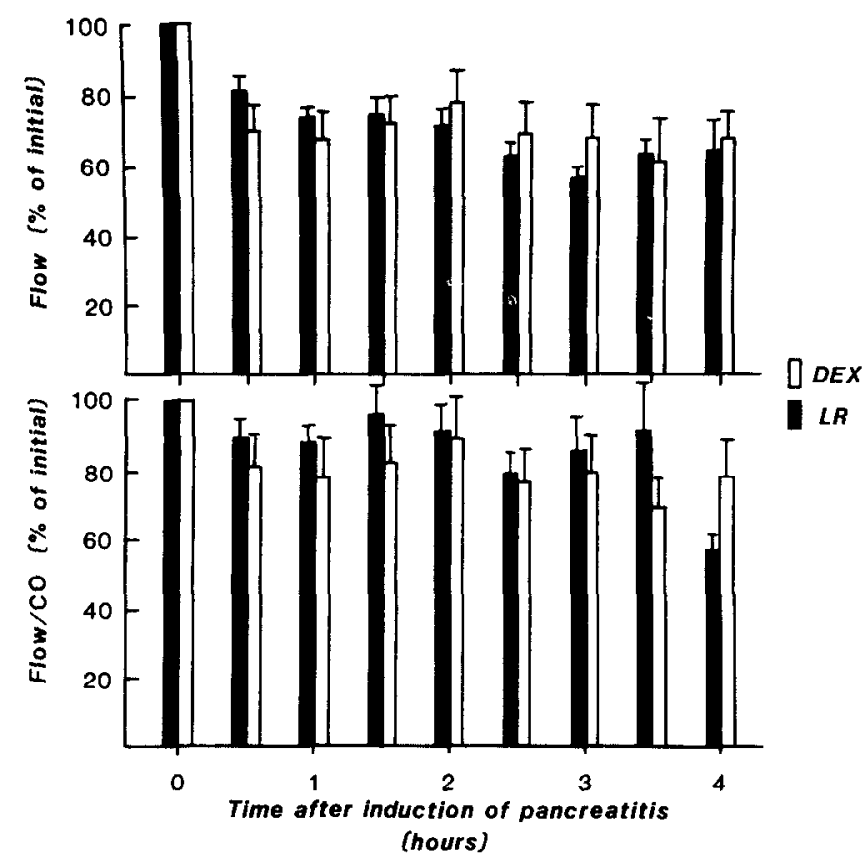

FIG. 5. Percent changes in pancreatic blood flow and in pancreatic flow as a fraction of cardiac output. Each dog served as its own control.

suggest that the predominant contribution to compromised pancreatic hemodynamics in acute pancreatitis is at the level of the pancreas.
Effective nutrient blood flow may also decrease as a result of increased AV shunting in acute pancreatitis. Papp, using a bile-induced pancreatitis model in dogs, demonstrated poor

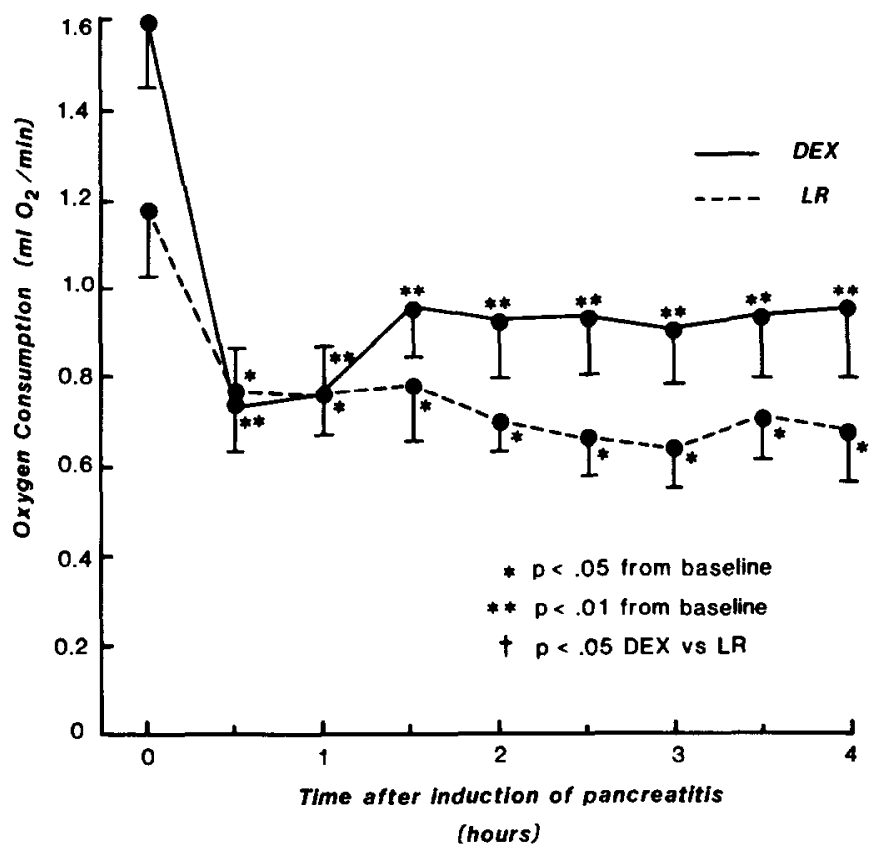

FIG. 6. Pancreatic oxygen consumption following induction of pancreatitis. 
capillary filling at three hr and an increase in both the size and number of AV shunts at 24 hr [24]. Donaldson and Schenk showed a decrease in pancreatic oxygen consumption associated with acute pancreatitis and hypothesized an increase in AV shunting to explain the apparent paradox of a decreased oxygen consumption despite decreased total pancreatic blood flow [9]. Although in the present study the percentage of total pancreatic blood flow passing through AV shunts increased following the induction of acute pancreatitis, the actual flow rate through AV shunts remained remarkably constant at $1-2 \mathrm{ml} / \mathrm{min}$ (Fig. 4). The decrease in pancreatic capillary flow closely paralleled changes in total pancreatic blood flow.

Altered cellular metabolism may also be important in the progression of pancreatitis. Decreased blood flow accompanied by increased oxygen extraction has been demonstrated in hypovolemic and cardiogenic shock states [30]. Paradoxically, the decrease in total pancreatic blood flow is associated with a decrease in pancreatic oxygen extraction in acute hemorrhagic pancreatitis $[6,9,20]$. Our present data confirm that a fall in pancreatic oxygen consumption occurs after the induction of acute pancreatitis, with a sharp decrease (35 and 54\% in LR and DEX groups, respectively) observed during the first $30 \mathrm{~min}$, followed by a relative plateau at a decreased level (Fig. 6). The causes for this decrease in pancreatic oxygen consumption are not apparent. The pattern of decreased oxygen consumption does not parallel the decline in pancreatic blood flow, and is not associated with an increase in arteriovenous shunt flow. Therefore, other possibilities must be entertained. A shift in the oxygen-hemoglobin affinity has been shown in acute pancreatitis [17], but a significant shift is unlikely in our study because of observed increases in systemic oxygen extraction (Fig. 3). An attractive hypothesis is that the initial insult causes metabolic damage to the pancreatic cell, with subsequent inability to utilize oxygen normally. Similar local metabolic changes in oxygen consumption have been noted in septic shock [31].

Low molecular weight dextran has been proposed as a therapeutic agent to prevent or reverse some of the microcirculatory and metabolic changes in the acutely inflamed pancreas. Initial trials of dextrans in acute experimental pancreatitis used clinical dextran, similar in molecular weight to dextran 70 , and hoped to utilize its volume-expanding effects [19]. Subsequently, rheologic and antithrombutic properties were described for LMW dextran which made it seem an ideal substance for use in pancreatitis. Gelin described decreased sludging of blood in small lumen vessels, in low-flow states associated with inflammatory processes, following infusion of LMW dextran [13]. The antithrombotic effect of LMW dextran should decrease formation of the microthrombi which have been demonstrated in low-flow states [18]. Both properties would theoretically help to improve pancreatic microcirculatory flow in acute pancreatitis. Experimentally, LMW dextran has been found to improve pancreatic blood flow in acute pancreatitis $[6,8,15]$. Further support for the use of LMW dextran comes from experimental studies in which decreased pancreatic oxygen consumption resulting from acute pancreatitis is partially normalized by the administration of LMW dextran $[6,8]$. Improved pancreatic blood flow has been proposed as the mechanism of action of this agent [8].

To date, evidence that increasing pancreatic blood flow will ameliorate the course of acute pancreatitis is all indirect. In one study, lysis of iatrogenically induced thrombi in the superior pancreaticoduodenal vein using streptokinase resulted in markedly improved survival; presumably clot lysis restored normal pancreatic venous outflow [14]. In another study, vasopressin administered to dogs with bile-trypsin-induced pancreatitis surprisingly increased survival [4]. It was subsequently shown that vasopressin causes increased pancreatic blood flow in experimental hemorrhagic pancreatitis [26]. Postganglionic sympathectomy has also been found to increase survival in dog models of acute hemorrhagic pancreatitis presumably by increasing pancreatic blood flow [16]. In the first published trial of LMW dextran in acute pan- 
creatitis, Anderson and Lewis were unable to demonstrate any significant improvement in survival [2]. However, other investigators subsequently observed improved survival rates with LMW dextran $[7,32]$, even if the infusion was initiated as late as $12 \mathrm{hr}$ after induction of pancreatitis [32]. Nevertheless, the data do not show directly that increases in pancreatic blood flow rather than other factors led to the increased survival rates.

In contrast to this body of evidence, our data do not support the use of LMW dextran in acute pancreatitis. Compared to infusion of lactated Ringer's solution alone, administration of LMW dextran changed none of the measured parameters significantly, including blood pressure, CVP, cardiac index, pancreatic blood flow, or pancreatic oxygen consumption. Trends were observed, but despite large numbers of animals in each group, no significant advantages resulting from the use of LMW dextran were observed.

Why then are the results found with LMW dextran in our study different from those of other investigators? Several possibilities exist. First, the volume effects of LMW dextran may play a role. Plasma volume deficits of $35-45 \%$ have been measured at $6 \mathrm{hr}$ in animals with untreated acute pancreatitis [19]. When administered as a bolus, LMW dextran (10\% solution) has been shown to increase plasma volume equal to the amount infused [12]. In contrast, crystalloid infusion increases plasma volume much less than the volume administered [12]. Critical evaluation of all experimental trials of LMW dextran in acute pancreatitis shows that in no case was an adequate volume of fluid administered to control animals. Our preliminary studies of canine acute pancreatitis showed that at least $6.5 \mathrm{ml} /$ $\mathrm{kg} / \mathrm{hr}$ of crystalloid was required to maintain blood pressure and CVP. However, the maximum fluid administered to controls in other published trials of LMW dextran was the equivalent of $2.0 \mathrm{ml} / \mathrm{kg} / \mathrm{hr}$ of crystalloid or $1.25 \mathrm{ml} / \mathrm{kg} / \mathrm{hr}$ of albumin. In none of those studies can the effect of LMW dextran on pancreatic blood flow be separated from its systemic volume effects. Because of large supplemental fluid replacement, in our series the volume effect of LMW dextran was minimized.

A second consideration is that of LMW dextran dosage. There are no available doseresponse data for the rheologic effects of LMW dextran. The dosage used has ranged from 0.2 $\mathrm{g} / \mathrm{kg}$ [11] to $1.5 \mathrm{~g} / \mathrm{kg}$ [6], often administered as a bolus. The dose used in this study $(0.15$ $\mathrm{g} / \mathrm{kg} / \mathrm{hr}$ as a continuous infusion) was similar to that used by Donaldson et al. [8]. Although renal excretion of a bolus of LMW dextran is half complete at $4 \mathrm{hr}$ [5], rapid excretion involves only the smaller molecules. The larger molecular weight fraction is excreted very slowly [12]. Therefore, administration of LMW dextran by continuous infusion results in an initially low but progressively rising blood level. This is in contrast to the initially high but thereafter decreasing LMW dextran level associated with bolus infusion. The effects of dosage and method of administration may be important in determining the efficacy of LMW dextran, but further studies will be required to clearly elucidate the influences of those variables.

LMW dextran may affect a multitude of other factors. Many trials have utilized survival as the end point, without attempting to control for systemic factors. The dramatic fall in arterial blood pressure seen by Donaldson et al. following the induction of acute pancreatitis was reproducible and severe $(25 \%$ reduction) in all experimental animals [8]. Shock leads to a host of additional systemic changes, as well as possible pancreatic changes. In the face of an increasing number of variables, the effect of LMW dextran on a single variable is more difficult to assess.

Finally, the time factor may be important. What happens with the administration of LMW dextran beyond $4 \mathrm{hr}$ from the induction of acute pancreatitis cannot be determined from our data.

\section{CONCLUSIONS}

1. Total pancreatic blood flow decreases significantly after induction of bile-trypsin-induced acute pancreatitis in the dog. The decreases in nutrient capillary flow alone ac- 
count for the overall flow reduction. There is no change in flow through AV shunts.

2. Pancreatic oxygen consumption falls early in the course of acute pancreatitis. The observed decrease despite reduced total pancreatic blood flow and unchanged AV shunt flow suggests that early metabolic damage to the cell may be responsible.

3. Low molecular weight dextran administered intravenously at a dose of $1.5 \mathrm{ml} / \mathrm{kg}$ / $\mathrm{hr}$ produces no significant improvement in pancreatic microcirculatory dynamics or in oxygen consumption in early acute pancreatitis.

\section{REFERENCES}

1. Anderson, M. C. Venous stasis in the transition of edematous pancreatitis to pancreatic necrosis. $J A M A$ 183: 534, 1963.

2. Anderson, M. C., and Lewis, M. B. Low-molecularweight dextran therapy in experimental pancreatitis. JAMA 192: 138, 1965.

3. Anderson, M. C., Schoenfeld, F. B., et al. Circulatory changes in acute pancreatitis. Surg. Clin. North Amer. 47: 127, 1967.

4. Andreadis, P., Kirlakou, K., et al. Vasopressin in the treatment of acute experimental pancreatitis. Ann. Surg. 166: 913, 1967.

5. Arturson, G., Granath, F., et al. The renal excretion of low molecular weight dextran. Acta Chir. Scand. 127: 543, 1964.

6. Becker, H., Senninger, N., et al. Dextran-40 and somatostatin in the treatment of acute experimental pancreatitis. News. Nat. Pancreatic Cancer Project 6: 7, 1981 (Abstract).

7. Carey, L. C. Low molecular weight dextran in experimental pancreatitis. Amer. J. Surg. 119: 197, 1970.

8. Donaldson, L. A., Williams, R. W., et al. Experimental pancreatitis: Effect of plasma and dextran on pancreatic blood flow. Surgery 84: 313, 1978.

9. Donaldson, L. A., and Schenk, W. G., Jr. Experimental acute pancreatitis: The changes in pancreatic oxygen consumption and the effect of dextran 40 . Ann. Surg. 190: 728, 1979.

10. Elliott, D. W. Treatment of acute pancreatitis with albumin and whole blood. Arch. Surg. 75: 573, 1957.

11. Gelin, L. E. Rheologic disturbances and the use of low viscosity dextran in surgery. Rev. Surg. 19: 385, 1962.

12. Gelin, L. E., Solvell, L., et al. The plasma volume expanding effect of low viscous dextran and macrodex. Acta Chir. Scand. 122: 309, 1961.

13. Gelin, L. E., and Thoren, O. K. A. Influence of low viscous dextran on peripheral circulation in man, a plethysmographic study. Acta Chir. Scand. 122: 303, 1961 .
14. Gerber, P. U., Meyer, W. H., Jr., et al. Experimental pancreatitis and fibrinolysin. Amer. Surg. 28: 445, 1962.

15. Goodhead, B. Acute pancreatitis and pancreatic blood flow. Surg. Gynecol. Obstet. 129: 331, 1969.

16. Goodhead, B., and Wright, P. W. The effect of postganglionic sympathectomy on the development of hemorrhagic pancreatitis in the dog. Ann. Surg. 170: 951, 1969.

17. Greenburg, A. G., Terlizzi, L., et al. Oxyhemoglobin affinity in acute pancreatitis. J. Surg. Res. 22: 561 , 1977.

18. Gruber, U. F. Dextran and the prevention of postoperative thromboembolic complications. Surg. Clin. N. Amer. 55: 679, 1975.

19. Hara, M., Thompson, B. W., et al. The comparative effect of dextran and blood in lethal sterile hemorrhagic pancreatitis. Surg. Forum 6: 377, 1955.

20. Knol, J. A., Eckhauser, F. E., et al. Hemodynamic alterations in experimental acute pancreatitis. Clin. Res. 29: 712A, 1981 (Abstract).

21. Musa, B. E., Nelson, A. W., et al. A model to study acute pancreatitis in the dog. J. Surg. Res. 21: 51, 1976.

22. Panum, P. L. Experimentelle Beitrage zur lehre von der Embolie. Virchows Arch. [Pathol. Anat.] 25: 308, 1862.

23. Papp, M., Makara, G. B., et al. A quantitative study of pancreatic blood flow in experimental pancreatitis. Gastroenterology 51: 524, 1966.

24. Papp, M., Ungvari, G. Y, et al. The effect of bileinduced pancreatitis on the intrapancreatic vascular pattern in dogs. Scand. J. Gastroenterol. 4: 681, 1969.

25. Pfeffer, R. B., Lazzarini-Robertson, A., Jr., et al. Gradations of pancreatitis, edematous, through hemorrhagic, experimentally produced by controlled injection of microspheres into blood vessels in dogs. Surgery 51: 761, 1962.

26. Pissiotis, C. A., Condon, R. E., et al. Effect of vasopressin on pancreatic blood flow in acute hemorrhagic pancreatitis. Amer. J. Surg. 123: 203, 1972.

27. Popper, H. L., Necheles, H., et al. Transition of pancreatic edema into pancreatic necrosis. Surg. Gynecol. Obstet. 87: 79, 1948.

28. Rich, A. R., and Duff, G. L. Experimental and pathological studies on the pathogenesis of acute hemorrhagic pancreatitis. Johns Hopkins Med. Bull. 58: 212, 1936.

29. Schiller, W. R., and Anderson, M. C. Microcirculation of the normal and inflamed canine pancreas. Ann. Surg. 181: 466, 1975.

30. Siegel, J. H., Farrell, E. J., et al. The surgical implications of physiologic patterns in myocardial infarction shock. Surgery 72: 126, 1972.

31. Wright, C. J., Duff, J. H., et al. Regional capillary blood flow and oxygen uptake in severe sepsis. Surg. Gynecol. Obstet. 132: 637, 1971.

32. Wright, R. W., and Goodhead, B. The value of dextrans in the treatment of experimental pancreatitis. Surgery 67: 807, 1970. 